\title{
BIFURCATION THEOREMS FOR HAMMERSTEIN NONLINEAR INTEGRAL EQUATIONS
}

\author{
FRANCESCA FARACI \\ Department of Mathematics \& Computer Science, University of Catania, \\ Viale A.Doria, 6, 95125 Catania, Italy \\ e-mail:ffaraci@dmi.unict.it
}

(Received 12 February, 2001; accepted 30 April, 2001)

\begin{abstract}
In this paper, we establish two results assuring that $\lambda=0$ is a bifurcation point in $L^{\infty}(\Omega)$ for the Hammerstein integral equation

$$
u(x)=\lambda \int_{\Omega} k(x, y) f(y, u(y)) d y .
$$
\end{abstract}

We also present an application to the two-point boundary value problem

$$
\left\{\begin{array}{l}
-u^{\prime \prime}=\lambda f(x, u) \quad \text { a.e. in }[0,1] \\
u(0)=u(1)=0
\end{array}\right.
$$

2000 Mathematics Subject Classification. 45G10, 47H30.

1. Introduction. Here and in the sequel, $\Omega \subset \mathbf{R}^{N}$ is a compact set, $k: \Omega \times \Omega \rightarrow \mathbf{R}$ is a measurable function, $f: \Omega \times \mathbf{R} \rightarrow \mathbf{R}$ is a Caratheódory function, with $f(x, 0)=0$ for all $x \in \Omega, \lambda$ is a real number.

Consider the Hammerstein integral equation

$$
u(x)=\lambda \int_{\Omega} k(x, y) f(y, u(y)) d y
$$

in the space $L^{\infty}(\Omega)$.

As usual, a $\lambda_{0} \in \mathbf{R}$ is said to be a bifurcation point for (1) in $L^{\infty}(\Omega)$ if $\left(0, \lambda_{0}\right)$ belongs to the closure in $L^{\infty}(\Omega) \times \mathbf{R}$ of the set

$$
\left\{(u, \lambda) \in L^{\infty}(\Omega) \times \mathbf{R}: u \text { solves (1) a.e. in } \Omega, u \neq 0\right\} .
$$

The most classical bifurcation result for (1) is certainly that due to M. A. Krasnosel'skii ([4], p. 342). In the case when the derivative $f_{y}^{\prime}(x, 0)$ exists, it is finite and one has

$$
\lim _{h \rightarrow 0} \frac{f(x, h)-h f_{y}^{\prime}(x, 0)}{h}=0
$$

uniformly with respect to $x \in \Omega$, such a result ensures (under further mild assumptions) that, for each nonzero eigenvalue $\mu$ of the linear integral operator 
$u \rightarrow \int_{\Omega} k(\cdot, y) f_{y}^{\prime}(y, 0) u(y) d y, \lambda=\frac{1}{\mu}$ is a bifurcation point for $(1)$ in $L^{\infty}(\Omega)$. Note that this result, on one hand, says nothing on the fact that also $\lambda=0$ can be a bifurcation point for (1), and, on the other hand, it has no sense in the case when the derivative $f_{y}^{\prime}(x, 0)$ does not exist or it is not finite.

More generally, although many other bifurcation theorems for (1) appeared after [4] (see, for instance, [1], [2], [3] and the references therein), it seems that there is no known result ensuring that $\lambda=0$ is a bifurcation point for $(1)$ in $L^{\infty}(\Omega)$.

The aim of the present paper is just to fill this gap by establishing Theorems 2.1 and 2.2, in the next section. It is worth noticing that our main assumption on the function $f$, when it does not depend on $x$, is

$$
\lim _{\xi \rightarrow 0} \frac{\int_{0}^{\xi} f(y) d y}{\xi^{2}}=+\infty .
$$

Clearly this condition and the existence of finite $f^{\prime}(0)$ exclude each other.

Note that a sufficient condition for the validity of the above equality is that $f^{\prime}(0)=+\infty$.

Our approach is variational. In particular, we use a local minimum principle recently pointed out by $\mathrm{B}$. Ricceri ([5]). We also present an application to a twopoint boundary value problem.

2. Results. Our first result is as follows:

Theorem 2.1. Assume that $k \in L^{\infty}(\Omega \times \Omega)$, that it is symmetric and that

$$
\int_{\Omega \times \Omega} k(x, y) \varphi(x) \varphi(y) d x d y>0
$$

for all $\varphi \in L^{2}(\Omega) \backslash\{0\}$. Further suppose that:

1. there are two numbers $\delta>0$ and $q>1$, and a function $\psi \in L^{q}(\Omega)$ such that

$$
\begin{aligned}
& \sup _{|y| \leq \delta}|f(x, y)| \leq \psi(x), \\
& \inf _{|\xi| \leq \delta} \int_{0}^{\xi} f(x, y) d y \geq 0
\end{aligned}
$$

for almost every $x \in \Omega$;

2. one has

$$
\max \left\{\lim _{\xi \rightarrow 0+} \frac{\inf _{x \in \Omega} \int_{0}^{\xi} f(x, y) d y}{\xi^{2}}, \lim _{\xi \rightarrow 0-} \frac{\inf _{x \in \Omega} \int_{0}^{\xi} f(x, y) d y}{\xi^{2}}\right\}=+\infty .
$$

Then, there exists a $\lambda^{\star}>0$ such that, for every $\left.\lambda \in\right] 0, \lambda^{\star}[$, equation (1) has a solution $u_{\lambda} \in L^{\infty}(\Omega) \backslash\{0\}$ satisfying

$$
\limsup _{\lambda \rightarrow 0+} \frac{\left\|u_{\lambda}\right\|_{L^{\infty}(\Omega)}}{\lambda} \leq\|k\|_{L^{\infty}(\Omega \times \Omega)}\|\psi\|_{L^{1}(\Omega)} .
$$


Proof. First, consider the function $\tilde{f}: \Omega \times \mathbf{R} \rightarrow \mathbf{R}$, defined by

$$
\tilde{f}(x, y)= \begin{cases}f(x,-\delta) & \text { if } y<-\delta \\ f(x, y) & \text { if }|y| \leq \delta \\ f(x, \delta) & \text { if } y>\delta\end{cases}
$$

Of course, $\tilde{f}$ is a Carathéodory function and it satisfies the following inequality:

$$
\sup _{y \in \mathbf{R}}|\tilde{f}(x, y)| \leq \psi(x)
$$

for almost every $x \in \Omega$. If we put

$$
F(x, \xi)=\int_{0}^{\xi} \tilde{f}(x, y) d y
$$

we have

$$
|F(x, \xi)| \leq \psi(x)|\xi|
$$

for all $\xi \in \mathbf{R}$, for almost every $x \in \Omega$.

It is no restriction to suppose that $1<q<2$. Let $p=\frac{q}{q-1}$. For each $u \in L^{2}(\Omega)$, $x \in \Omega$, put

$$
\mathbf{K}(u)(x)=\int_{\Omega} k(x, y) u(y) d y .
$$

Then, $\mathbf{K}$ is a self-adjoint, completely continuous, positive definite linear operator from $L^{2}(\Omega)$ into $L^{p}(\Omega)$, and there exists a completely continuous linear operator $H: L^{2}(\Omega) \rightarrow L^{p}(\Omega)$ such that

$$
\mathbf{K}(u)=H\left(H^{\star}(u)\right)
$$

for all $u \in L^{2}(\Omega)$, where $H^{\star}: L^{q}(\Omega) \rightarrow L^{2}(\Omega)$ is the adjoint of $H$ (see [4], I, $\S 4$, Theorem 4.4).

Instead of (1) let us consider the integral equation

$$
u(x)=\lambda \int_{\Omega} k(x, y) \tilde{f}(y, u(y)) d y,
$$

that is, in operator form,

$$
u=\lambda \mathbf{K}(\tilde{\mathbf{f}}(u)),
$$

where $\tilde{\mathbf{f}}(u)(x)=\tilde{f}(x, u(x))$. Since $\tilde{\mathbf{f}}$ acts from $L^{p}(\Omega)$ into $L^{q}(\Omega)$, equation (3) is equivalent to

$$
v=\lambda H^{\star}(\tilde{\mathbf{f}}(H(v)))
$$

in the space $L^{2}(\Omega)$, in the sense that to each solution $v \in L^{2}(\Omega)$ of (4) there corresponds a solution $u=H(v) \in L^{p}(\Omega)$ of (3), and conversely, to each solution $u \in L^{p}(\Omega)$ of (3) we can associate a solution $v=H^{\star}(\tilde{\mathbf{f}}(u)) \in L^{2}(\Omega)$ of (4). 
Let us introduce some further notations. For each $u \in L^{2}(\Omega)$, put

$$
\Psi(u)=\frac{1}{2}\|u\|_{L^{2}(\Omega)}^{2}
$$

and

$$
\Phi(u)=-\int_{\Omega} F(x, H(u)(x)) d x .
$$

By assumption on $\tilde{f}$, it follows that the functional

$$
u \rightarrow \Psi(u)+\lambda \Phi(u)
$$

is differentiable (see [4], I, §5), the solutions of equation (4) being precisely the critical points of this functional.

We claim that $\Phi$ is sequentially weakly continuous in $L^{2}(\Omega)$. Let $\left\{v_{n}\right\} \subseteq L^{2}(\Omega)$ be weakly convergent to $v_{0}$. Since $H$ is completely continuous, we can assume without loss of generality that $\left\{H\left(v_{n}\right)\right\}$ converges strongly to $H\left(v_{0}\right)$ in $L^{p}(\Omega)$, otherwise we should pass to a subsequence. Thus, there exist a function $h \in L^{p}(\Omega)$ and a subsequence $\left\{H\left(v_{n_{k}}\right)\right\}$ such that

$$
\left|H\left(v_{n_{k}}\right)(x)\right| \leq h(x)
$$

and $\left\{H\left(v_{n_{k}}\right)(x)\right\}$ converges to $H\left(v_{0}\right)(x)$ for almost all $x \in \Omega$. Hence, we have that

$$
\lim _{k \rightarrow+\infty} F\left(x, H\left(v_{n_{k}}\right)(x)\right)=F\left(x, H\left(v_{0}\right)(x)\right),
$$

and by (2),

$$
\left|F\left(x, H\left(v_{n_{k}}\right)(x)\right)\right| \leq \psi(x) h(x)
$$

for almost all $x \in \Omega$, for all $k \in \mathbf{N}$. Since $\psi h \in L^{1}(\Omega)$, the Lebesgue dominated convergence theorem assures that

$$
\Phi\left(v_{0}\right)=\lim _{k \rightarrow+\infty} \Phi\left(v_{n_{k}}\right),
$$

as we claimed.

Then, we readily see that it is possible to apply Theorem 2.1 of [5] to the functionals $\Phi$ and $\Psi$ defined above, endowing $L^{2}(\Omega)$ with the weak topology. So, by that result, there exists a suitable constant $c>0$ such that for each $\mu>c$ the restriction of the functional $\Phi+\mu \Psi$ to the unit open ball in $L^{2}(\Omega)$ has a global minimum, say $v_{\mu}$. Fix $\mu>c$. We claim that $v_{\mu} \neq 0$.

Since $k$ is bounded,

$$
\text { ess } \sup _{x \in \Omega}|H(\varphi)(x)| \leq \sqrt{\|k\|_{L^{\infty}(\Omega \times \Omega)}}\|\varphi\|_{L^{2}(\Omega)}
$$

for all $\varphi \in L^{2}(\Omega)$, (see [4], p. 64).

Suppose

$$
\lim _{\xi \rightarrow 0+} \frac{\inf _{x \in \Omega} \int_{0}^{\xi} f(x, y) d y}{\xi^{2}}=+\infty .
$$


Now, we choose a function $v \in L^{2}(\Omega)$, such that the set

$$
A=\{x \in \Omega: H(v)(x)>0\}
$$

has a positive measure.

By our assumption, if

$$
\eta>\frac{1}{2} \frac{\mu\|v\|_{L^{2}(\Omega)}^{2}}{\int_{A}|H(v)(x)|^{2} d x},
$$

there exists a $0<\bar{\delta}<\delta$ such that

$$
\inf _{x \in \Omega} F(x, \xi) \geq \eta \xi^{2}
$$

for all $0<\xi<\bar{\delta}$.

Fix $\tau^{\star}$ satisfying

$$
0<\tau^{\star}<\frac{\bar{\delta}}{\sqrt{\|k\|_{L^{\infty}(\Omega \times \Omega)}}\|v\|_{L^{2}(\Omega)}} .
$$

If $x \in A$, and $0<\tau<\min \left\{\tau^{\star}, \frac{1}{\|v\|_{L^{2}(\Omega)}}\right\}$, we readily have

$$
F(x, H(\tau v)(x)) \geq \eta \tau^{2}|H(v)(x)|^{2}
$$

and so, by 1 .,

$$
\int_{\Omega} F(x, H(\tau v)(x)) d x \geq \int_{A} F(x, H(\tau v)(x)) d x \geq \eta \tau^{2} \int_{A}|H(v)(x)|^{2} d x .
$$

Finally,

$$
\frac{\Phi(\tau v)}{\Psi(\tau v)} \leq-2 \frac{\eta \int_{A}|H(\tau v)(x)|^{2} d x}{\|v\|_{L^{2}(\Omega)}}<-\mu .
$$

So, $\|\tau v\|_{L^{2}(\Omega)}<1$ and $\Phi(\tau v)+\mu \Psi(\tau v)<0$. Since $\Phi(0)+\mu \Psi(0)=0$, then necessarily, $v_{\mu} \neq 0$.

In particular $v_{\mu}$ is a critical point for the functional $\Phi+\mu \Psi$.

Let $\lambda \in] 0, \frac{1}{c}[$.

Hence, there is a function $v_{\frac{1}{\lambda}} \neq 0$, that is a solution of (4). Put

$$
u_{\lambda}=H\left(v_{\frac{1}{\lambda}}\right) .
$$

Then,

$$
u_{\lambda}(x)=\lambda \int_{\Omega} k(x, y) \tilde{f}\left(y, u_{\lambda}(y)\right) d y
$$

almost everywhere in $\Omega$.

We have

$$
\left\|u_{\lambda}\right\|_{L^{\infty}(\Omega)} \leq \lambda\|k\|_{L^{\infty}(\Omega \times \Omega)}\|\psi\|_{L^{1}(\Omega)},
$$


so, if we put

$$
\lambda^{\star}=\min \left\{\frac{1}{c}, \frac{\delta}{\|k\|_{L^{\infty}(\Omega \times \Omega)}\|\psi\|_{L^{1}(\Omega)}}\right\},
$$

then, for all $\lambda \in] 0, \lambda^{\star}\left[\right.$ there is a solution $u_{\lambda} \in L^{\infty}(\Omega) \backslash\{0\}$ of equation (1), satisfying

$$
\frac{\left\|u_{\lambda}\right\|_{L^{\infty}(\Omega)}}{\lambda} \leq\|k\|_{L^{\infty}(\Omega \times \Omega)}\|\psi\|_{L^{1}(\Omega)}
$$

from which

$$
\limsup _{\lambda \rightarrow 0+} \frac{\left\|u_{\lambda}\right\|_{L^{\infty}(\Omega)}}{\lambda} \leq\|k\|_{L^{\infty}(\Omega \times \Omega)}\|\psi\|_{L^{1}(\Omega)} .
$$

Analogously, if

$$
\lim _{\xi \rightarrow 0-} \frac{\inf _{x \in \Omega} \int_{0}^{\xi} f(x, y) d y}{\xi^{2}}=+\infty
$$

holds, then choose a function $u$ such that the set

$$
\{x \in \Omega: H(u)(x)<0\}
$$

has a positive measure, and work with $\tau<0$.

REMARK 2.1. In particular 0 is a bifurcation point for equation (1).

Theorem 2.2. Assume that $k \in L^{\infty}(\Omega \times \Omega)$, that it is symmetric, that $k \geq 0$ and that

$$
\int_{\Omega \times \Omega} k(x, y) \varphi(x) \varphi(y) d x d y>0
$$

for all $\varphi \in L^{2}(\Omega) \backslash\{0\}$. Further suppose that:

1. there are two numbers $\delta>0$ and $q>1$, and a function $\psi \in L^{q}(\Omega)$ such that

$$
\begin{gathered}
\sup _{|y| \leq \delta}|f(x, y)| \leq \psi(x), \\
\inf _{|y| \leq \delta} f(x, y) y \geq 0
\end{gathered}
$$

for almost every $x \in \Omega$;

2. there is a set $D \subset \Omega$ of positive measure such that

$$
\lim _{\xi \rightarrow 0} \frac{\inf _{x \in D} \int_{0}^{\xi} f(x, y) d y}{\xi^{2}}=+\infty .
$$

Then, there exists $a \lambda^{\star}>0$ such that, for every $\left.\lambda \in\right] 0, \lambda^{\star}[$, equation (1) has two solutions $u_{\lambda}, w_{\lambda} \in L^{\infty}(\Omega) \backslash\{0\}$, with $u_{\lambda} \geq 0, w_{\lambda} \leq 0$, satisfying 
$\limsup _{\lambda \rightarrow 0+} \frac{\max \left\{\left\|u_{\lambda}\right\|_{L^{\infty}(\Omega)},\left\|w_{\lambda}\right\|_{L^{\infty}(\Omega)}\right\}}{\lambda} \leq\|k\|_{L^{\infty}(\Omega \times \Omega)}\|\psi\|_{L^{1}(\Omega)}$.

Proof. Let us introduce some notations.

Let $f_{1}: \Omega \times \mathbf{R} \rightarrow \mathbf{R}$ be defined by

$$
f_{1}(x, y)= \begin{cases}0 & \text { if } y<0 \\ f(x, y) & \text { if } 0 \leq y \leq \delta \\ f(x, \delta) & \text { if } y>\delta\end{cases}
$$

and set

$$
F_{1}(x, \xi)=\int_{0}^{\xi} f_{1}(x, y) d y
$$

and

$$
\Phi_{1}(u)=-\int_{\Omega} F_{1}(x, H(u)(x)) d x .
$$

We proceed as in the proof of Theorem 2.1, working with the functionals $\Psi$ and $\Phi_{1}$, both sequentially weakly lower semicontinuous in $L^{2}(\Omega)$.

So, there exists a positive constant $c_{1}$ such that for every $\mu>c_{1}$, the restriction of the functional $\Phi_{1}+\mu \Psi$ to the unit open ball in $L^{2}(\Omega)$ has a global minimum, say $v_{\mu}$.

Fix $\mu>c_{1}$. We claim that $v_{\mu} \neq 0$.

Let

$$
A=\left\{x \in \Omega: \int_{\Omega} k(x, y) d y=0\right\} .
$$

Note that $A$ has measure zero.

Indeed, if $A$ has a positive measure, the function defined by

$$
\chi_{A}(x)= \begin{cases}1 & \text { if } x \in A \\ 0 & \text { if } x \in \Omega \backslash A\end{cases}
$$

belongs to $L^{2}(\Omega) \backslash\{0\}$.

Since $k \geq 0$ and $\int_{\Omega} k(x, y) d y=0$, for every $x \in A$, we have

$$
\left.\int_{\Omega} \chi_{A}(x)\left(\int_{\Omega} k(x, y) \chi_{A}(y) d y\right) d x=\int_{A}\left(\int_{A} k(x, y)\right) d y\right) d x=0,
$$

against the hypothesis.

So, the function $\mathbf{K}(1)$ is positive almost everywhere in $\Omega$.

Consequently, if we put $v=H^{\star}(1)$, the set

$$
B=\{x \in \Omega: H(v)(x)>0\}
$$

has the same measure as $\Omega$, and so the set $B \cap D$ has a positive measure. 
By Assumption 2, it follows that

$$
\lim _{\xi \rightarrow 0+} \frac{\inf _{x \in D} \int_{0}^{\xi} f(x, y) d y}{\xi^{2}}=+\infty
$$

and then, if

$$
\eta>\frac{1}{2} \frac{\mu\|v\|_{L^{2}(\Omega)}^{2}}{\int_{B \cap D}|H(v)(x)|^{2} d x},
$$

there exists a $0<\delta_{1}<\delta$ such that

$$
\inf _{x \in D} F_{1}(x, \xi) \geq \eta \xi^{2}
$$

for all $0<\xi<\delta_{1}$.

Let $\tau>0$ be sufficiently small. We have

$$
F_{1}(x, H(\tau v)(x)) \geq \eta \tau^{2}|H(v)(x)|^{2}
$$

for all $x \in B \cap D$, and so

$$
\int_{\Omega} F_{1}(x, H(\tau v)(x)) d x \geq \int_{B \cap D} F_{1}(x, H(\tau v)(x)) d x \geq \eta \tau^{2} \int_{B \cap D}|H(v)(x)|^{2} d x .
$$

This proves that for a suitable $\tau>0,\|\tau v\|<1$ and $\Phi_{1}(\tau v)+\mu \Psi(\tau v)<0$, that is $v_{\mu} \neq 0$. As in Theorem 2.1, for all $\left.\lambda \in\right] 0, \frac{1}{c_{1}}$ [ there exists a function $u_{\lambda} \neq 0$ such that $u_{\lambda} \in L^{2}(\Omega)$ and

$$
u_{\lambda}(x)=\lambda \int_{\Omega} k(x, y) f_{1}\left(y, u_{\lambda}(y)\right) d y
$$

for almost all $x \in \Omega$.

Let

$$
C=\left\{x \in \Omega: u_{\lambda}(x)<0\right\} .
$$

By the definition of $f_{1}$,

$$
u_{\lambda}(x)=\lambda \int_{\Omega \backslash C} k(x, y) f_{1}\left(y, u_{\lambda}(y)\right) d y,
$$

so $C$ can not have positive measure. Hence, $u_{\lambda}$ is a non negative function.

If we choose

$$
0<\lambda<\min \left\{\frac{1}{c_{1}}, \frac{\delta}{\|k\|_{L^{\infty}(\Omega \times \Omega)}\|\psi\|_{L^{1}(\Omega)}}\right\}
$$

then $u_{\lambda}$ is a non negative solution of equation $(1)$, in $L^{\infty}(\Omega) \backslash\{0\}$, satisfying

$$
\left\|u_{\lambda}\right\|_{L^{\infty}(\Omega)} \leq \lambda\|k\|_{L^{\infty}(\Omega \times \Omega)}\|\psi\|_{L^{1}(\Omega)} .
$$


In the same way we deduce the existence of a non positive solution of (1): let $f_{2}: \Omega \times \mathbf{R} \rightarrow \mathbf{R}$ be defined by

$$
f_{2}(x, y)= \begin{cases}f(x,-\delta) & \text { if } y<-\delta \\ f(x, y) & \text { if }-\delta \leq y \leq 0 \\ 0 & \text { if } y \geq 0\end{cases}
$$

and notice that 2 . implies that

$$
\lim _{\xi \rightarrow 0-} \frac{\inf _{x \in D} \int_{0}^{\xi} f(x, y) d y}{\xi^{2}}=+\infty
$$

There is a suitable constant $c_{2}$ such that for every

$$
0<\lambda<\min \left\{\frac{1}{c_{2}}, \frac{\delta}{\|k\|_{L^{\infty}(\Omega \times \Omega)}\|\psi\|_{L^{1}(\Omega)}}\right\}
$$

there exists a non positive solution $w_{\lambda} \in L^{\infty}(\Omega) \backslash\{0\}$ of equation (1), satisfying

$$
\left\|w_{\lambda}\right\|_{L^{\infty}(\Omega)} \leq \lambda\|k\|_{L^{\infty}(\Omega \times \Omega)}\|\psi\|_{L^{1}(\Omega)} .
$$

Consequently, our conclusion follows with

$$
\lambda^{\star}=\min \left\{\frac{1}{c_{1}}, \frac{1}{c_{2}}, \frac{\delta}{\|k\|_{L^{\infty}(\Omega \times \Omega)}\|\psi\|_{L^{1}(\Omega)}}\right\} .
$$

By the proofs of the theorems above, one also obtains the following. that

Theorem 2.3. Assume that $k \in L^{\infty}(\Omega \times \Omega)$, that it is symmetric, that $k \geq 0$ and

$$
\int_{\Omega \times \Omega} k(x, y) \varphi(x) \varphi(y) d x d y>0
$$

for all $\varphi \in L^{2}(\Omega) \backslash\{0\}$. Further suppose that:

1. there are two numbers $\delta>0$ and $q>1$, and a function $\psi \in L^{q}(\Omega)$ such that

$$
\begin{aligned}
& \sup _{0 \leq y \leq \delta}|f(x, y)| \leq \psi(x) \quad\left(\text { resp. } \sup _{-\delta \leq y \leq 0}|f(x, y)| \leq \psi(x)\right), \\
& \inf _{0 \leq \xi \leq \delta} \int_{0}^{\xi} f(x, y) d y \geq 0 \quad\left(\text { resp. } \inf _{-\delta \leq \xi \leq 0} \int_{0}^{\xi} f(x, y) d y \geq 0\right)
\end{aligned}
$$

for almost every $x \in \Omega$;

2. there is a set $D \subset \Omega$ of positive measure such that

$$
\lim _{\xi \rightarrow 0+} \frac{\inf _{x \in D} \int_{0}^{\xi} f(x, y) d y}{\xi^{2}}=+\infty \quad\left(\text { resp. } \lim _{\xi \rightarrow 0-} \frac{\inf _{x \in D} \int_{0}^{\xi} f(x, y) d y}{\xi^{2}}=+\infty\right) .
$$


Then, there exists a $\lambda^{\star}>0$ such that, for every $\left.\lambda \in\right] 0, \lambda^{\star}[$, equation (1) has a solution $u_{\lambda} \in L^{\infty}(\Omega) \backslash\{0\}$, satisfying

$$
\limsup _{\lambda \rightarrow 0+} \frac{\left\|u_{\lambda}\right\|_{L^{\infty}(\Omega)}}{\lambda} \leq\|k\|_{L^{\infty}(\Omega \times \Omega)}\|\psi\|_{L^{1}(\Omega)} .
$$

3. An application. We conclude this paper giving an application of the Theorem 2.3 to the Dirichlet problem

$$
\left\{\begin{array}{l}
-u^{\prime \prime}=\lambda f(x, u) \quad \text { a.e. in }[0,1] \\
u(0)=u(1)=0
\end{array}\right.
$$

Theorem 3.1. Let $f:[0,1] \times \mathbf{R} \rightarrow \mathbf{R}$ be a Caratheódory function, with $f(x, 0)=0$ for all $x \in[0,1]$. Further suppose that:

1. there are two numbers $\delta>0$ and $q>1$, and a function $\psi \in L^{q}([0,1])$ such that

$$
\begin{aligned}
& \sup _{0 \leq y \leq \delta}|f(x, y)| \leq \psi(x), \\
& \inf _{0 \leq \xi \leq \delta} \int_{0}^{\xi} f(x, y) d y \geq 0
\end{aligned}
$$

for almost every $x \in[0,1]$;

2. there is a set $D \subset[0,1]$ of positive measure such that

$$
\lim _{\xi \rightarrow 0+} \frac{\inf _{x \in D} \int_{0}^{\xi} f(x, y) d y}{\xi^{2}}=+\infty .
$$

Then, there exists a $\lambda^{\star}>0$ such that, for every $\left.\lambda \in\right] 0, \lambda^{\star}[$, there exists a solution $u_{\lambda} \in W^{2, q}(] 0,1[) \backslash\{0\}$ of $(5)$, with $u_{\lambda} \geq 0$, satisfying

$$
\limsup _{\lambda \rightarrow 0+} \frac{\left\|u_{\lambda}\right\|_{L^{\infty}([0,1])}}{\lambda} \leq\|\psi\|_{L^{1}([0,1])} .
$$

Proof. Put $k:[0,1] \times[0,1] \rightarrow \mathbf{R}$

$$
k(x, y)= \begin{cases}(1-y) x & \text { if } 0 \leq x \leq y \leq 1 \\ (1-x) y & \text { if } 0 \leq y \leq x \leq 1\end{cases}
$$

Let us introduce

$$
f_{1}(x, y)= \begin{cases}0 & \text { if } y<0 \\ f(x, y) & \text { if } 0 \leq y \leq \delta \\ f(x, \delta) & \text { if } y>\delta\end{cases}
$$

It is easy to verify that the Dirichlet problem

$$
\left\{\begin{array}{l}
-u^{\prime \prime}=\lambda f_{1}(x, u) \quad \text { a.e. in }[0,1] \\
u(0)=u(1)=0
\end{array}\right.
$$


is equivalent to the following Hammerstein equation, whose kernel is the Green function $k$ :

$$
u(x)=\lambda \int_{0}^{1} k(x, y) f_{1}(y, u(y)) d y
$$

in the sense that $u$ is a solution of (6) if and only if it is a solution of the integral equation above (see ([6]), pages 47-49, 54-56).

By a classical result, the operator $\mathbf{K}$ is positive definite, so the hypotheses on $k$ are all fulfilled. We can apply Theorem 2.3 to $f_{1}$, obtaining that there exists a positive number $\lambda^{\star}$ such that for every $\left.\lambda \in\right] 0, \lambda^{\star}\left[\right.$ there is a function $u_{\lambda} \neq 0$, which solves the Dirichlet problem (6). By the hypothesis we have also that $u_{\lambda} \in W^{2, q}(] 0,1[)$. It is obvious that $u_{\lambda} \geq 0$, and so $u_{\lambda}$ solves the Dirichlet problem (5).

REMARK 3.1. It is clear that if $f(x, y) \geq 0$ for all $0 \leq y \leq \delta$ then, by the strong maximum principle, we have that $u_{\lambda}>0$.

Acknowledgements. The author would like to thank Professor B. Ricceri for helpful discussions on the topic of the paper and Dr. V. Moroz for his clear suggestions.

\section{REFERENCES}

1. J. Appell, B. V. Moroz, A. Vignoli and P. P. Zabrejko, On the application of Kielhofer's bifurcation theorem to Hammerstein equation with potential nonlinearity, Boll. Un. Mat. Ital. B (7) 8 (1994), no. 4, 833-850.

2. J. Appell, P.P. Zabrejko, Bifurcation points of Hammerstein equations, Integral Equations Operator Theory, 16 (1993), no. 1, 15-37.

3. S.G. Hu, Positive bifurcation points for a class of Hammerstein integral equations, Math. Appl. 7 (1994), no. 2, 206-211.

4. M. A. Krasnosel'skii, Topological methods in the theory of nonlinear integral equations (Pergamon Press, 1964).

5. B. Ricceri, A general variational principle and some of its applications, J. Comput. Appl. Math. 113 (2000), 401-410.

6. I. Stakgold, Green's functions and boundary value problems (John Wiley \& Sons, New York, 1979). 